\title{
On the finite-dimensional PUA representations of the Shubnikov space groups
}

\author{
$P$ M van den Broek \\ Institute for Theoretical Physics, University of Nijmegen, Toernooiveld, Nijmegen, The \\ Netherlands
}

Received 9 June 1977

\begin{abstract}
The finite-dimensional PUA representations of the Shubnikov space groups are discussed using the method of generalised induction given by Shaw and Lever. In particular we derive expressions for the calculation of the little groups.
\end{abstract}

\section{Introduction}

The relevance of the projective unitary-anti-unitary (PUA) representations of symmetry groups of physical systems is by now well known. If one considers alectrons in a crystalline potential with an external electromagnetic field the occurring symmetry groups are Shubnikov space groups. Examples for these external electromagnetic fields occurring in the literature are a uniform magnetic field (Brown 1964, Zak 1964a, b, Overhof and Rössler 1968, Opechowski and Tam 1969, Tam 1969) and a uniform magnetic field together with a uniform electric field (Ashby and Miller 1965, Tam 1970). These authors however do not consider pUA representations of Shubnikov space groups (except Brown, who derived the PU representations of the group of translations which obey periodic boundary conditions); they construct invariance operator groups for the Hamiltonian operator. The PUA representations of the Shubnikov space groups correspond to a special class of UA representations of these invariance operator groups. The derivation of these UA representations of the invariance operator groups however is more complicated than the derivation of the PUA representations of the Shubnikov space groups and has been given only for a special class of invariance operator groups (Overhof and Rössler 1968, Opechowski and Tam 1969).

This paper will be devoted to the study of the finite-dimensional irreducible PUA representations of the Shubnikov space groups. Although infinite-dimensional irreducible PUA representations exist we note that if we apply periodic boundary conditions, as is usually done, the groups become finite, and therefore the irreducible PUA representations are finite-dimensional. However, we will not impose periodic boundary conditions, but consider only finite-dimensional PUA representations. Our main tool will be the method of generalised induction, given by Shaw and Lever (1974). In $\S 3$ we will describe this method briefly, making use of the decomposition of factor systems given in a previous paper (van den Broek 1976). 


\section{Preliminaries}

Let $G$ be a group and $G_{0}$ a subgroup of $G$ of index 1 or 2. A PUA representation of $G$ with respect to $G_{0}$ is a mapping $D$ from $G$ into the operators on some Hilbert space $\mathscr{H}$ such that:

(i) $D(g)$ is unitary if $g \in G_{0}$ and anti-unitary if $g \notin G_{0}$;

(ii) $D(g) D\left(g^{\prime}\right)=\sigma\left(g, g^{\prime}\right) D\left(g g^{\prime}\right)$ for all $g, g^{\prime} \in G$ for some mapping $\sigma: G \times G \rightarrow U(1)$;

(iii) $D(e)=I$, where $e$ is the identity of $G$ and $I$ is the identity operator on $\mathscr{H}$.

The mapping $\sigma$ is called a factor system of $G$ with respect to $G_{0}$ and satisfies

$$
\sigma(g, e)=\sigma(e, g)=1 \quad \forall g \in G
$$

and

$$
\sigma\left(g, g^{\prime}\right) \sigma\left(g g^{\prime}, g^{\prime \prime}\right)=\sigma\left(g, g^{\prime} g^{\prime \prime}\right) \sigma^{g}\left(g^{\prime}, g^{\prime \prime}\right) \quad \forall g, g^{\prime}, g^{\prime \prime} \in G
$$

where $\lambda^{g}$ is defined by

$$
\lambda^{g}= \begin{cases}\lambda & \text { if } g \in G_{0} \\ \lambda^{*} & \text { if } g \notin G_{0}\end{cases}
$$

and the asterisk denotes complex conjugation.

In the sequel we will drop the phrase 'with respect to ...' wherever it is clear which the appropriate subgroup of index 1 or 2 is.

A factor system $\sigma$ of $G$ is called trivial if it can be written as

$$
\sigma\left(g, g^{\prime}\right)=c(g) c^{g}\left(g^{\prime}\right) c^{-1}\left(g g^{\prime}\right) \quad \forall g, g^{\prime} \in G
$$

for some mapping $c: G \rightarrow U(1)$.

Two factor systems $\sigma$ and $\sigma^{\prime}$ are called equivalent if $\sigma \sigma^{-1}$ is trivial.

From now on we suppose that the Hilbert space $\mathscr{H}$ is finite-dimensional.

Let $\left\{e_{1}, \ldots, e_{n}\right\}$ be an orthonormal basis of $\mathscr{H}$. For each operator $D(g)$ a unitary $n \times n$ matrix $\mathscr{D}(g)$ is defined by $\mathscr{D}_{i j}(g)=\left(e_{i}, D(g) e_{j}\right)$. The matrices $\mathscr{D}(g)$ form a PUA matrix representation or PUAM representation of $G$ :

$$
\mathscr{D}(g) \mathscr{D}^{g}\left(g^{\prime}\right)=\sigma\left(g, g^{\prime}\right) \mathscr{D}\left(g g^{\prime}\right)
$$

where $\mathscr{D}^{\boldsymbol{g}}$ is defined by

$$
\mathscr{D}^{8}= \begin{cases}\mathscr{D} & \text { if } g \in G_{0} \\ \mathscr{D}^{*} & \text { if } g \notin G_{0} .\end{cases}
$$

A PUA representation $D$ of $G$ is reducible if there is a proper subspace $\mathscr{H}$ of $\mathscr{H}$ which is invariant under $D(G)$; otherwise $D$ is irreducible. Two pUA representations $D_{1}$ and $D_{2}$ of $G$ are equivalent if there exists a unitary matrix $S$ such that

$$
\mathscr{D}_{1}(g)=S^{-1} \mathscr{D}_{2}(g) S^{g} .
$$

In the sequel we shall identify equivalent PUA representations, and also identify a PUA representation with the corresponding PUAM representation.

To conclude this preliminary section let us remark that we only need to consider factor systems of finite order to obtain the finite-dimensional PUA representations of a group. To see this, take the determinant of both sides of equation (2.5)

$$
[\operatorname{det} \mathscr{D}(g)]\left[\operatorname{det} \mathscr{D}\left(g^{\prime}\right)\right]^{g}=\sigma^{n}\left(g, g^{\prime}\right)\left[\operatorname{det} \mathscr{D}\left(g g^{\prime}\right)\right]
$$

if $\mathscr{D}$ is $n$-dimensional; this means that $\sigma^{n}\left(g, g^{\prime}\right)$ is a trivial factor system. 


\section{Generalised induction}

In this section we will describe briefly the procedure of generalised induction given by Shaw and Lever (1974), with the help of the decomposition of factor systems given by the author (van den Broek 1976). Let $G$ be a group, $G_{0}$ a subgroup of $G$ of index 1 or 2 and $H$ an invariant subgroup of $G$ of finite index. Let $K$ be the quotient group $G / H$. The elements of $K$ are the cosets of $G$ with respect to $H$. Elements of $H$ will be denoted by $a, b, c, \ldots$ and elements of $K$ by $\alpha, \beta, \gamma, \ldots$.

In the case where $H \nsubseteq G$ we suppose that the coset representatives are chosen to lie within $G_{0}$. Then each element of $G$ can uniquely be written as $(a, \alpha)$ if we define

$$
(a, \alpha)=\operatorname{ar}(\alpha) \text {. }
$$

Let $a^{\alpha}$ be defined by

$$
a^{\alpha}=r(\alpha) a r^{-1}(\alpha)
$$

and the mapping $m: K \times K \rightarrow H_{0}$ by

$$
r(\alpha) r(\beta)=m(\alpha, \beta) r(\alpha \beta)
$$

where $H_{0}=H \cap G_{0}$.

The multiplication in $G$ is now given by

$$
(a, \alpha)(b, \beta)=\left(a b^{\alpha} m(\alpha, \beta), \alpha \beta\right) .
$$

In the following we shall write $\lambda^{a}, \lambda^{\alpha}, \mathscr{D}^{a}$ and $\mathscr{D}^{\alpha}$ for brevity if we mean $\lambda^{(a, \epsilon)}, \lambda^{(e, \alpha)}$, $\mathscr{D}^{(a, \epsilon)}$ and $\mathscr{D}^{(e, \alpha)}$ respectively (see equations (2.3) and (2.6)). Let $\sigma$ be a factor system of $G$ with respect to $G_{0}$. We may choose $\sigma$ from its equivalence class in such a way that

$$
\sigma((a, \alpha),(b, \beta))=\gamma\left(a, b^{\alpha}\right) \gamma\left(a b^{\alpha}, m(\alpha, \beta)\right) \nu^{a b}(\alpha, \beta) P^{a}(\alpha, b)
$$

where $\gamma$ is the restriction of $\sigma$ to $H \times H$ and $\nu$ and $P$ are mappings from, respectively, $K \times K$ and $K \times H$ to $U(1)$ given by $\nu(\alpha, \beta)=\sigma((e, \alpha),(e, \beta))$ and $P(\alpha, b)=\sigma((e, \alpha)$, $(b, \epsilon))$ (van den Broek 1976).

This decomposition for $\sigma$ holds if and only if $\sigma((a, \epsilon),(e, \beta))=1$ for all $a \in H$ and all $\beta \in K$.

It will be supposed that $\sigma$ is of finite order and that the irreducible PUA representations of $H$ with respect to $H_{0}$ with factor system $\gamma$ are known.

Let $\mathscr{D}$ be an irreducible PUA representation of $H$ with factor system $\gamma$. For each $\alpha \in K$ the irreducible PUA representation $\mathscr{D}_{\alpha}$ of $H$ is defined by

$$
\mathscr{D}_{\alpha}\left(a^{\alpha}\right)=\mathscr{D}^{\alpha}(a) \sigma\left(\left(a^{\alpha}, \epsilon\right),(e, \alpha)\right) \sigma^{*}((e, \alpha),(a, \epsilon))
$$

which can be put in the form

$$
\mathscr{D}_{\alpha}\left(a^{\alpha}\right)=\mathscr{D}^{\alpha}(a) P^{*}(\alpha, a)
$$

using equation (3.5).

$\mathscr{D}_{\alpha}$ also belongs to the factor system $\gamma$. The set $\left\{\mathscr{D}_{\alpha} \mid \alpha \in K\right\}$ is called the orbit of $\mathscr{D}$. The elements $\alpha \in K$ for which $\mathscr{D}$ and $\mathscr{D}_{\alpha}$ are equivalent form a subgroup $K_{0}$ of $K$. The little group $L$ of $\mathscr{D}$ is defined by

$$
L=\left\{(a, \alpha) \mid a \in H, \alpha \in K_{0}\right\} .
$$

$L$ is a subgroup of $G$ which contains $H$ but is not necessarily an invariant subgroup. An irreducible PUA representation $D$ of $L$ with factor system $\sigma \downarrow L \times L$ is called allowable 
if $D \downarrow H=n \mathscr{D}$ for some integer $n$, i.e. if the irreducible components of the restriction of $D$ to $H$ are all equal to $\mathscr{D}$. Now we shall define the induced PuA representation $D \uparrow G$ of the allowable PUA representation $D$ of $L$.

Let $g_{1}=e, g_{2}, \ldots, g_{p}$ be left coset representatives of $G$ with respect to $L$. Then $G=\sum_{i=1}^{p} g_{i} L$.

Let $\mathscr{H}^{\prime}$ be the representation space of $D$, let $\mathscr{H}_{i}=\mathscr{C}^{\prime}$ for $i=1, \ldots, p$ and let $K_{i}$ be a fixed mapping from $\mathscr{H}^{\prime}$ to $\mathscr{H}_{i}$ which is unitary if $g_{i} \in G_{0}$ and anti-unitary if $g_{i} \notin G_{0}$. The representation space of $D \uparrow G$ then is $\mathscr{H}=\sum_{i=1}^{p} \oplus \mathscr{H}_{i}$ and $(D \uparrow G)(g)$ is given by its action on $\mathscr{H}_{i}$ :

$$
(D \uparrow G)_{i}(g)=\sigma\left(g, g_{i}\right) \sigma^{*}\left(g_{j}, g_{j}^{-1} g g_{i}\right) K_{j} D\left(g_{j}^{-1} g g_{i}\right) K_{i}^{-1}
$$

where $j$ is such that $g_{j}^{-1} g g_{i} \in L$.

Another way of defining $D \uparrow G$ is in terms of matrices:

$$
(D \uparrow G)_{j, k s}(g)= \begin{cases}0 & \text { if } g_{j}^{-1} g g_{k} \notin L \\ \sigma\left(g, g_{k}\right) \sigma^{*}\left(g_{j}, g_{j}^{-1} g g_{k}\right) D_{t s}^{g_{1}}\left(g_{j}^{-1} g g_{k}\right) & \text { if } g_{j}^{-1} g g_{k} \in L\end{cases}
$$

The procedure of generalised induction now is as follows:

(i) divide the irreducible PUA representations of $H$ with factor system $\gamma$ into orbits and take from each orbit one element $\mathscr{D}$;

(ii) determine the little group $L$ of $\mathscr{D}$;

(iii) find the allowable PUA representations of $L$;

(iv) induce these allowable PUA representations to $G$.

In this way all irreducible finite-dimensional PUA representations with factor system $\sigma$ are obtained.

Let us finally call attention to step (iii) in the induction procedure. We restrict ourselves to the case where $H \cong G_{0}$. For any $\alpha \in K_{0}$ there exists a unitary operator $U(\alpha)$ such that

$$
\mathscr{D}(a)=U(\alpha) \mathscr{D}_{\alpha}(a) U^{-1}(\alpha) .
$$

Define $U(a, \alpha)=\mathscr{D}(a) U(\alpha)$ for all $(a, \alpha) \in L$. Then $U(a, \alpha)$ is a PUA representation of $L$ with a factor system $\omega$ which may be shown to be of the form

$$
\omega((a, \alpha),(b, \beta))=\gamma\left(a, b^{\alpha}\right) \gamma\left(a b^{\alpha}, m(\alpha, \beta)\right) \mu(\alpha, \beta) P(\alpha, b) .
$$

The factor system $(\sigma \downarrow(L \times L)) \omega^{*}$ of $L$ thus reduces to a factor $\operatorname{system}(\nu / \mu)(\alpha, \beta)$ of $K_{0}$. The irreducible allowable PUA representations of $L$ with factor system $\sigma \downarrow(L \times L)$ are now given by $U(a, \alpha) \otimes E(\alpha)$, where $E$ runs through the irreducible PUA representations of $K_{0}$ with factor system $\nu \mu^{*}$. So step (iii) of the generalised induction procedure in the case where $H \leqq G_{0}$ reduces to the computation of $U(\alpha), \mu(\alpha, \beta)$ and the irreducible PUA representations of $K_{0}$ with factor system $\nu \mu^{*}$.

\section{The PUA representations of the Shubnikov space groups of type I and III}

Let $G$ be a Shubnikov space group of type I or III, $H$ its subgroup of translations and $G_{0}$ its non-magnetic subgroup.

The Shubnikov point groups $K$ and $K_{0}$ are defined by $G / H$ and $G_{0} / H$ respectively. The identities of $H$ and $K$ will be denoted by $e$ and $E$ respectively. Elements of $G$ will 
be denoted by $(t, R)$ where $t \in H$ and $R \in K$. We define $(t, R)$ by its action on space-time:

$$
(t, R)(x ; t)=\left(R x+t+t_{R} ; \epsilon_{R} t\right)
$$

where $\epsilon_{R}$ is defined by

$$
\epsilon_{R}=\left\{\begin{aligned}
1 & \text { if } R \in K_{0} \\
-1 & \text { if } R \notin K_{0}
\end{aligned}\right.
$$

and $t_{R}$ is a fixed non-primitive translation associated with $R$. The multiplication of elements of $G$ is now given by

$$
(t, R)\left(t^{\prime}, R^{\prime}\right)=\left(t+R t^{\prime}+m\left(R, R^{\prime}\right), R R^{\prime}\right)
$$

where the mapping $\boldsymbol{m}: K \times K \rightarrow H$ is given by

$$
m\left(R, R^{\prime}\right)=t_{R}+R t_{R^{\prime}}-t_{R R^{\prime}}
$$

Let $t_{1}, t_{2}$ and $t_{3}$ be basic translations of $H$. Then each element $t$ of $H$ can be written as $t=n_{1} t_{1}+n_{2} t_{2}+n_{3} t_{3}$. In the sequel we shall identify $t$ with the column vector with entries $n_{1}, n_{2}$ and $n_{3}$. Moreover each element $R$ of $K$ is given by the $3 \times 3$ matrix which represents $R$ with respect to the basic vectors $t_{1}, t_{2}$ and $t_{3}$, which is also denoted by $R$.

Let $\sigma$ be a factor system of $G$. From a previous paper (van den Broek 1977, to be referred to as I) it follows that we can choose $\sigma$ from its equivalence class in such a way that

$$
\sigma\left((t, R),\left(t^{\prime}, R^{\prime}\right)\right)=\gamma\left(t, R t^{\prime}\right) \gamma\left(t+R t^{\prime}, m\left(R, R^{\prime}\right)\right) \nu\left(R, R^{\prime}\right) P\left(R, t^{\prime}\right)
$$

where

$$
\gamma\left(t, t^{\prime}\right)=\exp \left[-2 \pi \mathrm{i}\left(\boldsymbol{t}^{\mathrm{T}} A \boldsymbol{t}^{\prime}\right)\right] .
$$

Here $A$ is a real antisymmetric $3 \times 3$ matrix

$$
A=\left(\begin{array}{ccc}
0 & a_{3} & -a_{2} \\
-a_{3} & 0 & a_{1} \\
a_{2} & -a_{1} & 0
\end{array}\right)
$$

and $\boldsymbol{t}^{\mathrm{T}}$ denotes the transpose of $\boldsymbol{t}$.

Furthermore for each $R \in K$ there exists a symmetric $3 \times 3$ matrix $B^{R}$ with halfinteger elements and a column vector $k(R)$ such that

$$
\epsilon_{R} R^{-1 \mathrm{~T}} A R^{-1}-A=B^{R}(\bmod 1)
$$

and

$$
P(R, t)=\exp \left(\pi \mathrm{i} t^{\mathrm{T}} R^{\mathrm{T}} B^{R} R t\right) \exp (-2 \pi \mathrm{i} k(R), R t) .
$$

So the factor system $\sigma$ is given by the matrix $A$, the vectors $k(R)$, the matrices $B^{R}$ and the mapping $\nu: K \times K \rightarrow U(1)$. These quantities are obtained if one determines the factor systems of $G$ as is done in $I$ and are supposed to be known in the sequel.

At this point we should note that we can take advantage of the fact that we are still able to choose the basic translations in a convenient way. For, since the column vectors $t$ and the matrices $R$ depend on the choice of the basic translations, also the quantities that determine the factor $\sigma$ depend on this choice. Suppose we take another set of basic translations $\boldsymbol{u}_{1}, \boldsymbol{u}_{2}$ and $\boldsymbol{u}_{3}$. Then a translation represented by $\boldsymbol{t}$ with respect to the old set 
of basic translations will be represented by $t^{\prime}=S t$ with respect to the new set where $S$ is an integer matrix with det $S= \pm 1$. The matrices $R$ are transformed into $R^{\prime}=S R S^{-1}$. From equation (4.6) it follows that the matrix which determines $\gamma$ now becomes $A^{\prime}=S^{-1 \Gamma} A S^{-1}$. From the equations (4.7) and (4.8) it follows that $B^{R}$ and $k(R)$ change into $S^{-1 \mathrm{~T}} B^{R} S^{-1}$ and $S^{-1 \mathrm{~T}} k(R)$ respectively. Since $A$ is antisymmetric, $A^{\prime}$ is also antisymmetric and can be written as

$$
A^{\prime}=\left(\begin{array}{ccc}
0 & a_{3}^{\prime} & -a_{2}^{\prime} \\
-a_{3}^{\prime} & 0 & a_{1}^{\prime} \\
a_{2}^{\prime} & -a_{1}^{\prime} & 0
\end{array}\right) .
$$

We will show that the basic translations, and thus the matrix $S$, can be chosen in such a way that $a_{1}^{\prime}=a_{2}^{\prime}=0$. Let the vectors $a$ and $a^{\prime}$ be given by $\left(a_{1}, a_{2}, a_{3}\right)$ and $\left(a_{1}^{\prime}, a_{2}^{\prime}, a_{3}^{\prime}\right)$ respectively. Then from $A^{\prime}=S^{-1 \mathrm{~T}} A S^{-1}$ it follows $a^{\prime}=(\operatorname{det} S) S a$.

Since $\gamma$ is a factor system of finite order it follows that the entries of $a$ and $a^{\prime}$ are rational. Therefore we can find a vector $\boldsymbol{u}$ which is a multiple of $a$ and has integer entries which are relatively prime. Now we choose as new basic translation $u_{3}$ the translation which is represented by $u$ with respect to $t_{1}, t_{2}$ and $t_{3} ; \mathbf{u}_{1}$ and $\boldsymbol{u}_{2}$ are chosen arbitrarily. Now $S u=(0,0,1)$ and since $a^{\prime}$ is a multiple of $S u$ it follows $a_{1}^{\prime}=a_{2}^{\prime}=0$. Therefore in the sequel we may assume that the basic vectors are chosen in such a way that $a_{1}=a_{2}=0$. The factor system $\gamma$ of $H$ is then given by $a_{3}$ which is a rational number, so we can write $a_{3}=m / 2 N$ where $m$ and $N$ are integers with no common factor.

The PU representations of $H$ with factor system $\gamma$ may be determined with the generalised induction procedure, starting from a subgroup of $H$ where $\gamma$ is trivial, analogous to the derivation by Opechowski and Tam (1969) of the unitary vector representations of their invariance translation operator group. However, from the results of Opechowski and Tam the Pu representations of $H$ with factor system $\gamma$ may be read off immediately; they are all $N$ dimensional and labelled by a vector $\boldsymbol{k}$, the three components of which run through the interval $[0,1)$. Their matrix elements are given by

$$
\left[D^{k}(t)\right]_{i j}=\left\{\begin{array}{l}
\exp \left[2 \pi i\left(\frac{k_{1}}{N} t_{1}+\frac{k_{2}}{N} t_{2}+k_{3} t_{3}+\frac{k_{2}}{N}(j-i)-\frac{m}{N} t_{1}\left(\frac{1}{2} t_{2}+j\right)\right)\right] \\
\quad \text { if } \frac{t_{2}+j-i}{N} \text { is integer } \\
0 \quad \text { otherwise. }
\end{array}\right.
$$

The character $\chi^{k}(t)=\sum_{i=1}^{N}\left[D^{k}(t)\right]_{i i}$ is given by

$\chi^{k}(t)=\left\{N \exp \left[2 \pi \mathrm{i}\left(\frac{k_{1}}{N} t_{1}+\frac{k_{2}}{N} t_{2}+k_{3} t_{3}-\frac{m}{2 N} t_{1} t_{2}\right)\right] \quad\right.$ if both $\frac{t_{1}}{N}$ and $\frac{t_{2}}{N}$ are integer otherwise.

So the characters are different from zero only on the subgroup $H_{1}$ of $H$ of index $N^{2}$ given by

$$
H_{1}=\left\{t \in H \mid t_{1}=n_{1} N ; t_{2}=n_{2} N ; n_{1}, n_{2} \text { integer }\right\} .
$$

According to equation (3.6) we define for each $R \in K$

$$
D_{R}^{k}(t)=\left[D^{k}\left(R^{-1} t\right)\right]^{R} P^{*}\left(R, R^{-1} t\right) .
$$


The character $\chi_{R}^{k}(t)$ of $D_{R}^{k}(t)$ is given by

$$
\chi_{R}^{k}(t)=\left[\chi^{k}\left(R^{-1} t\right)\right]^{R} P^{*}\left(R, R^{-1} t\right) .
$$

$D_{R}^{k}$ and $D^{k}$ are equivalent if and only if $\chi_{R}^{k}=\chi^{k}$, or

$$
\chi^{k}(t) P\left(R, R^{-1} t\right)=\left[\chi^{k}\left(R^{-1} t\right)\right]^{R} \quad \forall t \in H .
$$

Now $H_{1}$ is invariant under the matrices $R$ of $K$. This can be seen as follows: from equation (4.7) we have

$$
\epsilon_{R}(\operatorname{det} R) R a=a \quad\left(\bmod \frac{1}{2}\right)
$$

if $a$ is the vector with entries $a_{1}, a_{2}$ and $a_{3}$. With $a_{1}=a_{2}=0$ and $a_{3}=m / 2 N$ it follows

$$
R\left(\begin{array}{l}
0 \\
0 \\
1
\end{array}\right)=\left(\begin{array}{l}
0 \\
0 \\
1
\end{array}\right) \quad(\bmod N)
$$

Therefore $R\left(\begin{array}{l}0 \\ 0 \\ 1\end{array}\right)$ belongs to $H_{1}$ and thus $R\left(\begin{array}{c}N t_{1} \\ N t_{2} \\ t_{3}\end{array}\right)$ also belongs to $H_{1}$. This means that we only need to consider equation (4.14) for $t \in H_{1}$. Let the matrix $Q$ be defined by

$$
Q=\left(\begin{array}{ccc}
N & 0 & 0 \\
0 & N & 0 \\
0 & 0 & 1
\end{array}\right)
$$

then the elements of $H_{1}$ can be written as $Q t$ where $t \in H$. The equations (4.14) and (4.10) now become respectively

$$
\chi^{k}(R O t) P(R, Q t)=\left[\chi^{k}(Q t)\right]^{R} \quad \forall t \in H
$$

and

$$
\chi^{k}(Q t)=N \exp \left[2 \pi \mathrm{i}\left(k \cdot t-\frac{1}{2} m N t_{1} t_{2}\right)\right] .
$$

Substituting the equations (4.19) and (4.8) in equation (4.18) gives

$$
\begin{aligned}
& \epsilon_{R} k \cdot \boldsymbol{t}+\boldsymbol{k}(R) \cdot R Q \boldsymbol{t}-\boldsymbol{k} \cdot Q^{-1} R Q t \\
& \quad=\frac{1}{2} \epsilon_{R} m N t_{1} t_{2}+\frac{1}{2} t^{\mathrm{T}} Q R^{\mathrm{T}} B^{R} R Q t-\frac{1}{2} m N\left(Q^{-1} R Q t\right)_{1}\left(Q^{-1} R Q t\right)_{2} \quad(\bmod 1)
\end{aligned}
$$

Let us denote the right-hand side of this equation by $f(t)$, then $f\left(t+t^{\prime}\right)-f(t)-f\left(t^{\prime}\right)=\frac{1}{2} \epsilon_{R} m N t^{\mathrm{T}} C t^{\prime}+t^{\mathrm{T}} Q R^{\mathrm{T}} B^{R} R Q t^{\prime}-\frac{1}{2} m N t^{\mathrm{T}} Q R^{\mathrm{T}} Q^{-1} C Q^{-1} R Q t^{\prime}$

where

$$
C=\left(\begin{array}{lll}
0 & 1 & 0 \\
1 & 0 & 0 \\
0 & 0 & 0
\end{array}\right)
$$

This can be written as

$$
f\left(\boldsymbol{t}+\boldsymbol{t}^{\prime}\right)-f(\boldsymbol{t})-f\left(\boldsymbol{t}^{\prime}\right)=\boldsymbol{t}^{\mathrm{T}} D \boldsymbol{t}^{\prime}
$$


where the matrix $D$ is given, if we use equation (4.7), by

$D=\frac{1}{2} \epsilon_{R} m N C-\frac{1}{2} m N Q R^{\mathrm{T}} Q^{-1} C Q^{-1} R K+\epsilon_{R} Q A Q-Q R^{\mathrm{T}} A R Q \quad(\bmod 1)$

or

$D=\epsilon_{R}\left(\frac{1}{2} m N C+Q A Q\right)-Q R^{\mathrm{T}} Q^{-1}\left(\frac{1}{2} m N C+Q A Q\right) Q^{-1} R Q \quad(\bmod 1)$.

But

$$
\frac{1}{2} m N C+Q A Q=\frac{1}{2} m N\left(\begin{array}{lll}
0 & 2 & 0 \\
0 & 0 & 0 \\
0 & 0 & 0
\end{array}\right) .
$$

So the matrix $D$ has only integer entries. This means that $f\left(t+t^{\prime}\right)-f(t)-f\left(t^{\prime}\right)$ is integer and therefore we may write

$$
f(t)=I(R) . t \quad(\bmod 1) .
$$

From the definition of $f(t)$ it follows that we can choose $l(R)$ by

$$
\begin{aligned}
{[l(R)]_{i} } & =\frac{1}{2}\left[Q R^{\mathrm{T}} B^{R} R Q\right]_{i i}-\frac{1}{2} m N\left(Q^{-1} R Q\right)_{1 i}\left(Q^{-1} R Q\right)_{2 i} \\
& =\frac{1}{2} Q_{i i}^{2}\left[R^{\mathrm{T}} B^{R} R\right]_{i i}-\frac{m}{2 N} Q_{i i}^{2} R_{1 i} R_{2 i} .
\end{aligned}
$$

Equation (4.20) now becomes

$$
\epsilon_{R} k \cdot t+k(R) \cdot R Q t-k \cdot Q^{-1} R Q t=l(R) \cdot t(\bmod 1) .
$$

and we finally obtain

$$
\epsilon_{R} k+Q R^{\mathrm{T}} k(R)-O R^{\mathrm{T}} Q^{-1} k-l(R)=0 \quad(\bmod 1) .
$$

So $D^{k}$ and $D_{R}^{k}$ are equivalent if and only if equation (4.29) holds. This gives us the orbit and the little group of $D^{k}$, and therefore the finite-dimensional PUA representations of $G$ are obtained with the generalised induction procedure.

\section{The PUA representations of the Shubnikov space groups of type II}

Let $\boldsymbol{G}$ be a Shubnikov space group of type II and $G$ its unitary subgroup which is a type I Shubnikov space group. The notation of the elements of $G$ will be as in the previous section. Elements of $\boldsymbol{G}$ will be denoted by $(t, R, \alpha)$ where $(t, R) \in G, \alpha \in C_{2}=\{e, T\}$ and $T$ denotes the time-reversal operator.

We define $(t, R, \alpha)$ by $(t, R, e)=(t, R)$ and $(t, R, T)=(t, R) T$.

Let $\omega$ be a factor system of $\boldsymbol{G}$. From I it follows that $\omega$ may be chosen from its equivalence class in such a way that

$$
\omega\left((t, R, \alpha),\left(t^{\prime}, R^{\prime}, \alpha^{\prime}\right)\right)=\sigma\left((t, R),\left(t^{\prime}, R^{\prime}\right)\right) N\left(\alpha, \alpha^{\prime}\right) M\left(\alpha,\left(t^{\prime}, R^{\prime}\right)\right)
$$

where $\sigma$ is a factor system of $G$ with the properties described in the previous section and $N$ and $M$ are mappings from, respectively, $C_{2} \times C_{2}$ and $C_{2} \times G$ into $U(1)$ with the properties

$$
\begin{aligned}
& N(e, e)=N(e, T)=N(T, e)=M(T,(e, E))=1 \\
& M(e,(t, R))=1 \quad \forall(t, R) \in G
\end{aligned}
$$


and

$$
\begin{aligned}
M\left(T,(t, R)\left(t^{\prime}, R^{\prime}\right)\right) & \\
& =M(T,(t, R)) M\left(T,\left(t^{\prime}, R^{\prime}\right)\right) \sigma^{2}\left((t, R),\left(t^{\prime}, R^{\prime}\right)\right) \quad \forall(t, R),\left(t^{\prime}, R^{\prime}\right) \in G .
\end{aligned}
$$

Since $H$ is an invariant subgroup of $\boldsymbol{G}$ and $\omega((t, E, e),(e, R, \alpha))=1$ we may also decompose $\omega$ as follows

$\omega\left((t, R, \alpha),\left(t^{\prime}, R^{\prime}, \alpha^{\prime}\right)\right)$

$$
=\gamma\left(\boldsymbol{t}, \boldsymbol{t}^{\prime(R, \alpha)}\right) \gamma\left(\boldsymbol{t}+\boldsymbol{t}^{(R, \alpha)}, \boldsymbol{u}\left((R, \alpha),\left(R^{\prime}, \alpha^{\prime}\right)\right)\right) \mu\left((R, \alpha),\left(R^{\prime}, \alpha^{\prime}\right)\right) P^{\prime}\left((R, \alpha), \boldsymbol{t}^{\prime}\right)
$$

where $(R, \alpha)$ stands for the coset with representative $(\boldsymbol{e}, R, \alpha)$. Here $\boldsymbol{t}^{(R, \alpha)}$ equals $R \boldsymbol{t}$ and $\boldsymbol{u}\left((R, \alpha),\left(R^{\prime}, \alpha^{\prime}\right)\right)$ is equal to $\boldsymbol{m}\left(R, R^{\prime}\right)$. From the equations (5.1), (5.2) and (4.5) we obtain

$$
P^{\prime}((R, \alpha), t)=P(R, t) M(\alpha,(t, E)) .
$$

Now for each irreducible Pu representation $D^{k}$ of $H$ with factor system $\gamma$ we define $D_{(R, \alpha)}^{k}$ by

$$
D_{(R, \alpha)}^{k}(t)=\left[D^{k}(t)\right]^{\alpha} P^{*}(R, t) M^{*}(\alpha,(t, E))
$$

Reasoning along the same lines as in the previous section it follows that $D^{k}$ and $D_{(R, \alpha)}^{k}$ are equivalent if and only if

$$
\chi^{k}(R Q t) P(R, Q t) M(\alpha,(Q t, E))=\left[\chi^{k}(Q t)\right]^{\alpha} .
$$

From the equations (5.4) and (4.5) it follows

$$
M\left(T, t+t^{\prime}\right)=M(T, t) M\left(T, t^{\prime}\right) \gamma^{2}\left(t, t^{\prime}\right)
$$

So $\gamma^{2}$ is a trivial factor system of $H$, and therefore $a_{3}=0$ or $\frac{1}{4}$, so $m / N=0$ or $\frac{1}{2}$. Since now $\gamma^{2}\left(\boldsymbol{t}, \boldsymbol{t}^{\prime}\right)=\exp \left(2 \pi \mathrm{i} \frac{m}{N}\left(t_{1} t_{2}^{\prime}-t_{2} t_{1}^{\prime}\right)\right)=\exp \left(2 \pi \mathrm{i} \frac{m}{N}\left[t_{1} t_{2}+t_{1}^{\prime} t_{2}^{\prime}-\left(t_{1}+t_{1}^{\prime}\right)\left(t_{2}+t_{2}^{\prime}\right)\right]\right)$

we can write $M(T, t)$ as

$$
M(T, t)=\exp \left(2 \pi i \frac{m}{N} t_{1} t_{2}\right) \exp (2 \pi i k(T) \cdot t)
$$

for some vector $k(T)$ which is determined by the factor system $\omega$. Substituting the equations (4.19), (4.8) and (5.11) in equation (5.8) gives

$$
\begin{aligned}
\epsilon(\alpha) \boldsymbol{k} \cdot \boldsymbol{t}-\boldsymbol{k} & \cdot Q^{-1} R Q \boldsymbol{t}+\boldsymbol{k}(R) \cdot R Q \boldsymbol{t}-\boldsymbol{k}(\alpha) \cdot Q \boldsymbol{t} \\
& =\frac{1}{2} m N t_{1} t_{2}+\frac{1}{2} \boldsymbol{t}^{\mathrm{T}} Q R^{T} B^{R} R Q \boldsymbol{t}-\frac{1}{2} m N\left(Q^{-1} R Q \boldsymbol{t}\right)_{1}\left(Q^{-1} R Q t\right)_{2}(\bmod 1)
\end{aligned}
$$

Here $\epsilon(\alpha)$ is given by $\epsilon(e)=1$ and $\epsilon(T)=-1 ; \boldsymbol{k}(\alpha)$ is given by $\boldsymbol{k}(T)$ and the zero vector $\boldsymbol{k}(e)$. Just as in the previous section the right-hand side of equation (5.12) is equal to $l(R) . t(\bmod 1)$ where $l(R)$ is given by equation $(4.27)$. We finally obtain

$$
\boldsymbol{\epsilon}(\alpha) \boldsymbol{k}-Q R^{\mathrm{T}} Q^{-1} \boldsymbol{k}+Q R^{\mathrm{T}} \boldsymbol{k}(R)-Q \boldsymbol{k}(\alpha)-\boldsymbol{l}(R)=0 \quad(\bmod 1)
$$

as the equation which determines the orbit and the little group of $D^{k}$. 


\section{The PUA representations of the Shubnikov space groups of type IV}

Let $\boldsymbol{G}$ be a Shubnikov space group of type IV, $G$ its unitary subgroup, which is a type I Shubnikov space group, and let $\boldsymbol{H}$ be the black and white lattice of $G$. $\boldsymbol{H}$ is given by $H$, which is again the subgroup of translations of $G$, and an extra translation $t_{0}$, with $2 t_{0} \in H$, which occurs in $G$ in combination with the time-reversal operator. The notation of the elements of $G$ will be as in the preceding sections. Elements of $G$ will be denoted by $(t, R, \alpha)$ where $(t, R) \in G$ and $\alpha \in C_{2}=\{e, a\}$ with $a^{2}=e$. We define $(t, R, \alpha)$ by $(t, R, e)=(t, R)$ and $(t, R, a)=\left(t+R t_{0}, R\right) T$ where $\left(t+R t_{0}, R\right)$ acts on space vectors in the usual way and $T$ denotes again the time-reversal operator.

Let $\omega$ be a factor system of $\boldsymbol{G}$. From I it follows that $\omega$ may be chosen from its equivalence class in such a way that

$\omega\left((t, R, \alpha),\left(t^{\prime}, R^{\prime}, \alpha^{\prime}\right)\right)$

$$
=\sigma\left((t, R),\left(t^{\prime}, R^{\prime}\right)^{\alpha}\right) \sigma\left((t, R)\left(t^{\prime}, R^{\prime}\right)^{\alpha}, n\left(\alpha, \alpha^{\prime}\right)\right) N\left(\alpha, \alpha^{\prime}\right) M\left(\alpha,\left(t^{\prime}, R^{\prime}\right)\right)
$$

where $\sigma$ is a factor system of $G$ with the properties described in $\S 4,(t, R)^{\alpha}$ is given by $(t, R)^{e}=(t, R)$ and $(t, R)^{a}=\left(t+t_{0}-R t_{0}, R\right)$, and $n\left(\alpha, \alpha^{\prime}\right)$ is given by $n(e, e)=n(e, a)=$ $n(a, e)=(e, E)$ and $n(a, a)=\left(2 t_{0}, E\right)$. Furthermore the mappings $N$ and $M$ from $C_{2} \times C_{2}$ and $C_{2} \times G$ respectively into $U(1)$ have the following properties:

$$
\begin{aligned}
& N(e, e)=N(e, a)=N(a, e)=M(a,(e, E))=1 \\
& P(e,(t, R))=1 \quad \forall(t, R) \in G
\end{aligned}
$$

and

$M\left(a,(t, R)\left(t^{\prime}, R^{\prime}\right)\right)=M(a,(t, R)) M\left(a,\left(t^{\prime}, R^{\prime}\right)\right) \sigma\left((t, R)^{a},\left(t^{\prime}, R^{\prime}\right)^{a}\right) \sigma\left((t, R),\left(t^{\prime}, R^{\prime}\right)\right)$.

Just as in the previous section we obtain equation (5.5), the only difference being that here $\boldsymbol{u}$ is given by

$$
\boldsymbol{u}\left((R, \alpha),\left(R^{\prime}, \alpha^{\prime}\right)\right)=\boldsymbol{m}\left(R, R^{\prime}\right)+\delta(\alpha) R\left[t_{0}-\epsilon\left(\alpha^{\prime}\right) R^{\prime} t_{0}\right] .
$$

Here $\delta$ and $\epsilon$ are given by $\delta(e)=0, \delta(a)=1, \epsilon(e)=1$ and $\epsilon(T)=-1$. Also equation (5.6) is valid here which means that $D_{(R, \alpha)}^{k}$ again is given by equation (5.7). From the equations (6.4) and (4.5) it follows

$$
M\left(a, t+t^{\prime}\right)=M(a, t) M\left(a, t^{\prime}\right) \gamma^{2}\left(t, t^{\prime}\right) .
$$

So the situation is completely analogous to the situation in the previous section; we can write

$$
M(\alpha, t)=\exp \left(2 \pi \mathrm{i} \frac{m}{N} t_{1} t_{2}\right) \exp (2 \pi \mathrm{i} \boldsymbol{k}(\alpha) \cdot t)
$$

and the final result is that $D^{k}$ and $D_{(R, \alpha)}^{k}$ are equivalent if and only if

$$
\epsilon(\alpha) \boldsymbol{k}-Q R^{\mathrm{T}} Q^{-1} \boldsymbol{k}+Q R^{\mathrm{T}} \boldsymbol{k}(R)-Q \boldsymbol{k}(\alpha)-\boldsymbol{l}(R)=0 \quad(\bmod 1) .
$$

\section{Acknowledgment}

I thank Dr $\mathrm{T}$ Janssen for reading the manuscript. 


\section{References}

Ashby N and Miller S C 1965 Phys. Rev. A 139 428-36

Broek P M van den 1976 J. Phys. A: Math. Gen $9855-62$

1977 J. Phys. A: Math. Gen. 10 649-58

Brown E 1964 Phys. Rev. A 133 1038-44

Opechowski W and Tam W G 1969 Physica 42 529-56

Overhof H and Rössler U 1968 Phys. Stat. Solidi 26 461-8

Shaw R and Lever J 1974 Commun. Math. Phys. 38 257-77

Tam W G 1969 Physica 42 557-64

1970 Physica 46 469-74

Zak J 1964a Phys. Rev. A 134 1602-6

_ 1964b Phys. Rev. A 134 1607-11 